\title{
Impact of climate change on Indian agriculture: a review
}

\author{
R. K. Mall • Ranjeet Singh • Akhilesh Gupta • \\ G. Srinivasan $\cdot$ L. S. Rathore
}

\section{Erratum to: Climatic Change (2006) 78: 445-478 DOI 10.1007/s10584-005-9042-x}

The original article was published without Tables I, II, III, IV, V, and VI, shown here on the following pages.

The online version of the original article can be found at doi:10.1007/s10584-005-9042-x.

R. K. Mall $(\bowtie)$

Central Ground Water Board,

New Delhi 110001, India

e-mail: mall_raj@rediffmail.com

R. Singh $\cdot$ A. Gupta $\cdot$ L. S. Rathore

National Center for Medium Range Weather Forecasting,

Noida 201307, India

G. Srinivasan

India Meteorological Department,

Lodi Road, New Delhi 110003, India

Present address:

R. K. Mall

Department of Geophysics, Banaras Hindu University,

Varanasi 221005, India 
TABLE I Selective reports on observed climate change during the twentieth century over India

\begin{tabular}{|c|c|c|c|}
\hline Region & Temperature & Rainfall & Reference \\
\hline All India & $\begin{array}{l}\text { Increase in } 0.4^{\circ} \mathrm{C} / 100 \text { years } \\
\text { in the mean annual } \\
\text { temperature. }\end{array}$ & & $\begin{array}{l}\text { Hingane et al. } \\
1985\end{array}$ \\
\hline All India & $\begin{array}{l}\text { Increase in max. Temp. } \\
\left(0.6^{\circ} \mathrm{C} / 100 \mathrm{yrs}\right) \\
\text { Min temp trend less. } \\
\text { General increase in the } \\
\text { diurnal range of temp }\end{array}$ & & $\begin{array}{l}\text { Rupa Kumar et al. } \\
1994\end{array}$ \\
\hline Western Himalayas & $\begin{array}{l}\text { Winter season, Srinagar, } \\
\text { Mussoorie and Mukteswar } \\
\text { shows increasing trend } \\
\left(0.5^{\circ} \mathrm{C} / 100 \text { year }\right) \\
\text { Monsoon season, Srinagar, } \\
\text { which is beyond the } \\
\text { monsoon regime, shows } \\
\text { significant increasing } \\
\text { trend, whereas Mussoorie } \\
\text { and Dehradun which are } \\
\text { at the foothills of } \\
\text { Himalaya show } \\
\text { decreasing trend }\end{array}$ & $\begin{array}{l}\text { No increasing or decreasing } \\
\text { trend for last } 100 \text { years }\end{array}$ & Pant et al. 1999 \\
\hline \multirow[t]{2}{*}{$\begin{array}{l}\text { Indo-Gangetic Plain } \\
\text { Region (IGPR) }\end{array}$} & $\begin{array}{l}\text { Annual surface air } \\
\text { temperature of the IGPR } \\
\text { showed rising trend }\left(0.53^{\circ} \mathrm{C} /\right. \\
100 \text {-year, during } 1875- \\
1958\end{array}$ & $\begin{array}{l}\text { Summer monsoon rainfall } \\
\text { over western IGPR shows } \\
\text { increasing trend }(170 \mathrm{~mm} / \\
100 \text { years) from } 1900 \text { while } \\
\text { over central IGPR it shows } \\
\text { decreasing trend ( } 5 \mathrm{~mm} / \\
100 \text { years) from } 1939 \text { and } \\
\text { over eastern IGPR } \\
\text { decreasing trend }(50 \mathrm{~mm} / 100 \\
\text { years) during } 1900-1984 \text { and } \\
\text { increasing trend }(480 \mathrm{~mm} / 100 \\
\text { years) during } 1984-1999 .\end{array}$ & $\begin{array}{l}\text { Singh and } \\
\text { Sontakke } 2002\end{array}$ \\
\hline & $\begin{array}{l}\text { Decreasing trend } \\
\left(-0.93^{\circ} \mathrm{C} / 100 \text {-year during }\right. \\
1958-1997\end{array}$ & $\begin{array}{l}\text { Westward shift in rainfall } \\
\text { activities over the IGPR. }\end{array}$ & \\
\hline All-India & & $\begin{array}{l}\text { Monsoon rainfall is trend } \\
\text { less and is mainly random in } \\
\text { nature over a long period }\end{array}$ & $\begin{array}{l}\text { Mooley and } \\
\text { Parthasarathy } \\
\text { 1984; Thapliyal } \\
\text { and Kulshrestha } \\
\text { 1991 }\end{array}$ \\
\hline $\begin{array}{l}\text { NE Peninsula, NE } \\
\text { India, NW } \\
\text { Peninsula, West } \\
\text { Coast and Central } \\
\text { Peninsula }\end{array}$ & & $\begin{array}{l}\text { NE peninsula, NE India and } \\
\text { NW peninsula show } \\
\text { decreasing trend in the Indian } \\
\text { summer monsoon rainfall ( }-6 \\
\text { to } 8 \% \text { of normal/100 years) } \\
\text { while increasing trend was } \\
\text { noticed along the west coast } \\
\text { and over central peninsula }\end{array}$ & $\begin{array}{l}\text { Rupa Kumar et al. } \\
1992\end{array}$ \\
\hline
\end{tabular}


TABLE I (continued)

\begin{tabular}{|c|c|c|c|}
\hline Region & Temperature & Rainfall & Reference \\
\hline & & $\begin{array}{l}\text { ( }+10 \text { to } 12 \% \text { of normal/ } \\
100 \text { years })\end{array}$ & \\
\hline $\begin{array}{l}\text { Western and Eastern } \\
\text { Himalayas }\end{array}$ & & $\begin{array}{l}\text { Western Himalayas get more } \\
\text { snowfall than the eastern } \\
\text { Himalayas during winter. } \\
\text { More rainfall in the eastern } \\
\text { Himalayas than in the western } \\
\text { Himalayas during the } \\
\text { monsoon season }\end{array}$ & $\begin{array}{l}\text { Kripalani et al. } \\
1996\end{array}$ \\
\hline Rajasthan desert & & $\begin{array}{l}\text { Slight increase in monsoon } \\
\text { rainfall; in spite of large } \\
\text { inter annual variations }\end{array}$ & $\begin{array}{l}\text { Pant and Hingne } \\
1998\end{array}$ \\
\hline \multirow[t]{2}{*}{$\begin{array}{l}\text { Luni River Basin } \\
\text { (Arid west } \\
\text { Rajasthan) }\end{array}$} & $\begin{array}{l}\text { Rising trend at } \\
\text { Barmer, Jodhpur, } \\
\text { Ajmer and Pali }\end{array}$ & $\begin{array}{l}\text { Annual rainfall indicating } \\
\text { increasing tendency at } 19 \\
\text { station (around Ajmer in } \\
\text { upper part of the Luni basin }\end{array}$ & Singh et al. 2001 \\
\hline & $\begin{array}{l}\text { Decreasing treand } \\
\text { at Udaipur and } \\
\text { jwaibandh }\end{array}$ & $\begin{array}{l}\text { Decreasing trend at the } \\
\text { remaining nine stations in } \\
\text { lower Luni basin i.e. Barmer. }\end{array}$ & \\
\hline
\end{tabular}


TABLE II Selective reports on projected climate change during next century over India

\begin{tabular}{|c|c|c|c|}
\hline Region & Temperature & Rainfall & Reference \\
\hline $\begin{array}{l}\text { All } \\
\text { India }\end{array}$ & $\begin{array}{l}\text { Increase in winter or rabi crop growing } \\
\text { season temperature by } 1-4^{\circ} \mathrm{C} \text { with } \\
\text { increased } \mathrm{CO}_{2} \text { concentration }\end{array}$ & $\begin{array}{l}\text { Precipitation increase of } \\
\text { approximately } 20 \% \text {. } \\
\text { Increase in specific } \\
\text { humidity by } 19 \% \\
\text { Increase in heavy rainfall days } \\
\text { during the summer } \\
\text { monsoon period } \\
\text { i.e. in kharif crop growing season, } \\
\text { and an increased inter annual } \\
\text { variability }\end{array}$ & $\begin{array}{l}\text { Bhaskaran } \\
1995\end{array}$ \\
\hline $\begin{array}{l}\text { All } \\
\text { India }\end{array}$ & $\begin{array}{l}\text { Average temperature change is predicted to } \\
\text { be in the range of } 2.33-4.78^{\circ} \mathrm{C} \text { with a } \\
\text { doubling in } \mathrm{CO}_{2} \text { concentration }\end{array}$ & $\begin{array}{l}\text { Increase in the frequency of heavy } \\
\text { rainfall events }\end{array}$ & $\begin{array}{l}\text { Lonergan } \\
1998\end{array}$ \\
\hline $\begin{array}{l}\text { All } \\
\text { India }\end{array}$ & $\begin{array}{l}\text { Increase in annual mean day and night } \\
\text { temperature of } 0.7 \text { and } 1.0^{\circ} \mathrm{C} \text { in } 2040 \mathrm{~s} \\
\text { with respect to } 1980 \mathrm{~s} \text {. }\end{array}$ & & $\begin{array}{l}\text { Lal et al. } \\
1995\end{array}$ \\
\hline $\begin{array}{l}\text { All } \\
\text { India }\end{array}$ & Increase in mean temp. $\left({ }^{\circ} \mathrm{C}\right)$ : & Change in rainfall $(\%)$ & $\begin{array}{l}\text { Lal et al. } \\
2001\end{array}$ \\
\hline & $\begin{array}{l}\text { Year } 2020 \mathrm{~s} \\
\text { Kharif season, } 0.87 \text { to } 1.12 \\
\text { Rabi season, } 1.08 \text { to } 1.54 \\
\text { Year } 2050 \mathrm{~s} \\
\text { Kharif season, } 1.81 \text { to } 2.37 \\
\text { Rabi season, } 2.54 \text { to } 3.18 \\
\text { Year } 2080 \mathrm{~s} \\
\text { Kharif season, } 2.91 \text { to } 4.62 \\
\text { Rabi season, } 4.14 \text { to } 6.31\end{array}$ & $\begin{array}{l}\text { Year } 2020 \mathrm{~s} \\
\text { Kharif season, } 1.8 \text { to } 5.1 \\
\text { Rabi season, }-2.0 \text { to } 4.7 \\
\text { Year } 2050 \mathrm{~s} \\
\text { Kharif season, } 7.2 \text { to } 10.5 \\
\text { Rabi season, }-9.2 \text { to } 3.8 \\
\text { Year } 2080 \mathrm{~s} \\
\text { Kharif season, } 10.1 \text { to } 15.2 \\
\text { Rabi season, }-24.8 \text { to }-4.5\end{array}$ & \\
\hline $\begin{array}{l}\text { All } \\
\text { India }\end{array}$ & $\begin{array}{l}\text { Increase in annual mean temperature by } \\
1.3-1.7^{\circ} \mathrm{C}\end{array}$ & $\begin{array}{l}6-13 \% \text { increase in kharif season } \\
\text { rainfall }\end{array}$ & $\begin{array}{l}\text { Rupa } \\
\text { Kumar \& } \\
\text { Ashrit } \\
2001\end{array}$ \\
\hline \multirow[t]{3}{*}{$\begin{array}{l}\text { All } \\
\text { India }\end{array}$} & Year 2050s & Year 2050s & $\begin{array}{l}\text { Rupa } \\
\text { Kumar } \\
\text { et al. } \\
2003\end{array}$ \\
\hline & $\begin{array}{l}\text { South of } 25^{\circ} \mathrm{N} \text { (south of cities such as } \\
\text { Udaipur, Khajuraho and Varanasi) } \\
\text { maximum temp. will increase by } 2-4^{\circ} \mathrm{C} \text {. } \\
\text { In the northern region the increase in } \\
\text { maximum temp. by more than } 4^{\circ} \mathrm{C} \text {. }\end{array}$ & $\begin{array}{l}\text { Decrease in number of rainy days over } \\
\text { a major part of the country. This } \\
\text { decrease is more in western and } \\
\text { central part (by more than } 15 \text { days) }\end{array}$ & \\
\hline & $\begin{array}{l}\text { Increase in minimum temperature up to } 4^{\circ} \mathrm{C} \\
\text { all over the country. }\end{array}$ & $\begin{array}{l}\text { While near the foothills of Himalayas } \\
\text { (Uttaranchal state) and in northeast } \\
\text { India the number of rainy days } \\
\text { increase by } 5-10 \text { days. } \\
\text { Overall increase in the rainy day } \\
\text { intensity by } 1-4 \mathrm{~mm} / \text { day except for } \\
\text { small areas in the northwest India } \\
\text { where the rainfall intensities decrease } \\
\text { by } 1 \mathrm{~mm} / \text { day. }\end{array}$ & \\
\hline
\end{tabular}


TABLE III Grain yield ( $\mathrm{t} \mathrm{ha}^{-1}$ ) of wheat in current weather and percent change in response to climate change (425 pp $\mathrm{CO}_{2}, 2^{\circ} \mathrm{C}$ increase in mean temperature) in different regions of India

\begin{tabular}{|c|c|c|c|c|c|c|}
\hline \multirow[t]{2}{*}{ Region } & \multicolumn{2}{|c|}{ Potential yield } & \multicolumn{2}{|c|}{ Irrigated yields } & \multicolumn{2}{|c|}{ Rainfed yields } \\
\hline & Current & Percent $(\%)$ change & Current & Percent $(\%)$ change & Current & Percent $(\%)$ change \\
\hline$>27^{\circ} \mathrm{N}$ & 6.66 & -3.9 & 4.89 & 3.7 & 2.95 & 28.6 \\
\hline $25-27^{\circ} \mathrm{N}$ & 5.84 & -1.5 & 4.78 & -4.4 & 3.34 & -7.2 \\
\hline $23-25^{\circ} \mathrm{N}$ & 5.86 & -5.6 & 4.18 & -10.8 & 1.17 & -19.6 \\
\hline $20-23^{\circ} \mathrm{N}$ & 4.18 & -18.4 & 2.29 & -18.3 & 0.51 & -11.8 \\
\hline$<20^{\circ} \mathrm{N}$ & 3.69 & -17.3 & 2.43 & -21.4 & 0.97 & -23.9 \\
\hline
\end{tabular}

(Source: Aggarwal and Kalra 1994)

TABLE IV Increase in yield (\%) of different cultivars under modified climate of northwest India

\begin{tabular}{|c|c|c|c|c|}
\hline \multirow[t]{2}{*}{ Cultivar (Station) } & \multicolumn{2}{|c|}{ Rainfed } & \multicolumn{2}{|c|}{ Irrigated } \\
\hline & S-II & S-III & S-II & S-III \\
\hline WH542 (Hisar) & 37 & 30 & 27 & 23 \\
\hline HD2329 (Ludhiana) & 31 & 22 & 16 & 12 \\
\hline HD2285 (Delhi) & 29 & 24 & 22 & 19 \\
\hline Sonalika (Pantnagar) & 34 & 27 & 22 & 18 \\
\hline Raj3765 (Jaipur) & 36 & 29 & 28 & 22 \\
\hline
\end{tabular}

S-I: current climate; S-II: $T_{\max }+1.0^{\circ} \mathrm{C}, T_{\min }+1.5^{\circ} \mathrm{C}, 2 \times \mathrm{CO}_{2}$; S-III: $T_{\max }+2.0^{\circ} \mathrm{C}, T_{\min }+2.5^{\circ} \mathrm{C}, 2 \times \mathrm{CO}_{2}$.

(Source: Attri and Rathore, 2003) 


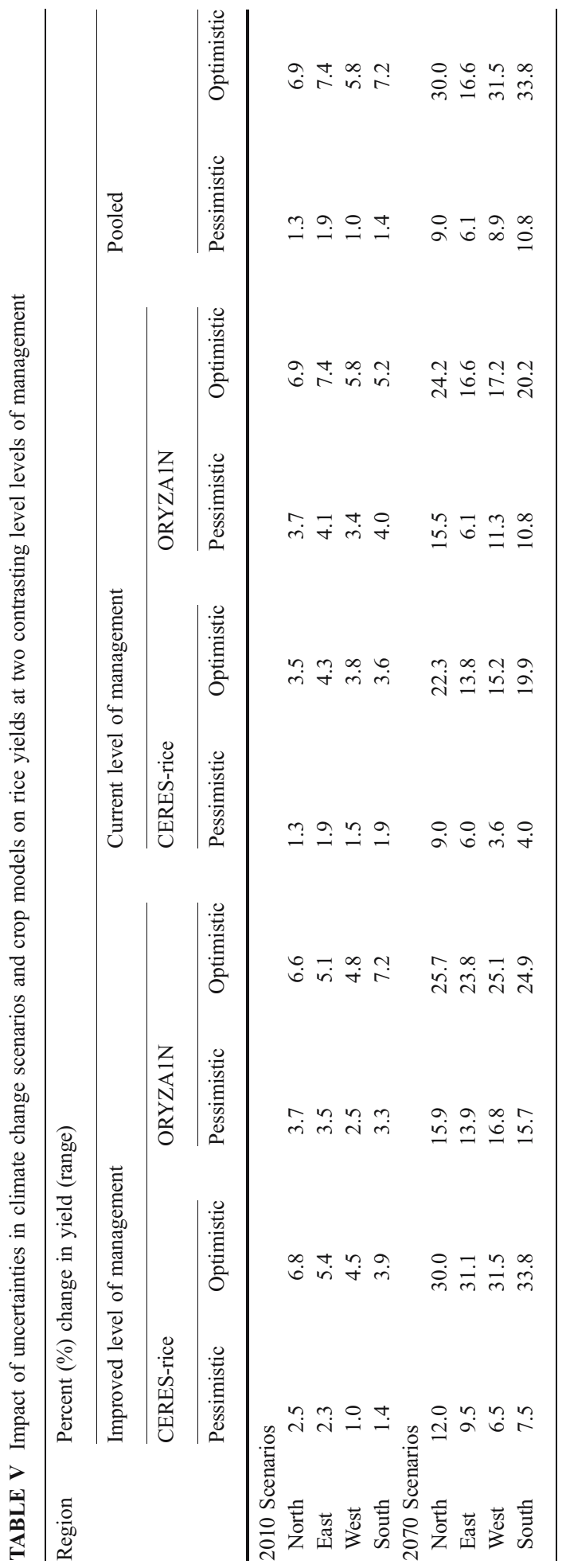


TABLE VI Selective reports on impacts of projected climate change on yield of different crops in India

\begin{tabular}{|c|c|c|c|}
\hline Crop & Region & Yield impact & Reference \\
\hline \multirow[t]{9}{*}{ Rice } & All India & Increase (region wise and scenarios based) & Aggarwal and Mall 2002 \\
\hline & $\begin{array}{l}\text { North-West, } \\
\text { Central }\end{array}$ & Increase (decrease in North-West) & Rathore et al. 2001 \\
\hline & South & Decrease/increase (scenarios based) & Sasendran et al. 1999 \\
\hline & North-West & Decrease/increase (scenarios based) & Lal et al. 1998 \\
\hline & Punjab & Decrease/increase (scenarios based) & Hundal and Kaur 1996 \\
\hline & All India & Increase & Mohandass et al. 1995 \\
\hline & North & Increase & Achanta 1993 \\
\hline & All India & Decrease & $\begin{array}{l}\text { Sinha and Swaminathan } \\
1991\end{array}$ \\
\hline & Northern India & Decrease & Saini \& Nanda 1986 \\
\hline \multirow[t]{9}{*}{ Wheat } & North & No impact/decrease (scenarios based) & Aggarwal 2003 \\
\hline & North-west & +16 to $+37 \%$ & Attri and Rathore 2003 \\
\hline & $\begin{array}{l}\text { North-West, } \\
\text { Central }\end{array}$ & $\begin{array}{l}\text { No effect in NW, } 10-15 \% \text { decrease in } \\
\text { Central }\end{array}$ & Aggarwal 2000 \\
\hline & North-west & Decrease/increase (scenarios based) & Lal et al. 1998 \\
\hline & Punjab & Decrease/increase (scenarios based) & Hundal and Kaur 1996 \\
\hline & All India & Decrease & $\begin{array}{l}\text { Gangadhar Rao and Sinha } \\
1994\end{array}$ \\
\hline & All India & $\begin{array}{l}\text { Decrease/increase (region wise and scenarios } \\
\text { based) }\end{array}$ & Aggarwal and Kalra 1994 \\
\hline & All India & $\begin{array}{l}\text { Decrease/increase (region wise and scenarios } \\
\text { based) }\end{array}$ & Aggarwal and Sinha 1993 \\
\hline & All India & Decrease & $\begin{array}{l}\text { Sinha and Swaminathan } \\
1991\end{array}$ \\
\hline \multirow[t]{2}{*}{ Soybean } & All India & $\begin{array}{l}\text { Decrease/increase (region wise and scenarios } \\
\text { based) }\end{array}$ & Mall et al. 2004 \\
\hline & Central & Decrease/increase (scenarios based) & Lal et al. 1999 \\
\hline \multirow[t]{2}{*}{ Maize } & North & $\begin{array}{l}\text { Decrease/increase (region wise and } \\
\text { scenarios based) }\end{array}$ & Sahoo 1999 \\
\hline & Punjab & Decrease & Hundal and Kaur 1996 \\
\hline Chickpea & All India & Decrease & Mandal 1998 \\
\hline Pigenpea & All India & Decrease & Mandal 1998 \\
\hline Groundnut & Punjab & Decrease & Hundal and Kaur 1996 \\
\hline \multirow[t]{2}{*}{ Sorghum } & All India & Decrease & Chatterjee 1998 \\
\hline & All India & $\begin{array}{l}\text { Decrease/increase (region wise and } \\
\text { scenarios based) }\end{array}$ & $\begin{array}{l}\text { Gangadhar Rao et al. } \\
1995\end{array}$ \\
\hline $\begin{array}{l}\text { Brassica } \\
\text { (Mustard) }\end{array}$ & North & Increase & Upreaty et al. 1996 \\
\hline
\end{tabular}

